\title{
Impact of Rotation Rate on Bismuth Saturation in GaAsBi Grown by Molecular Beam Epitaxy
}

\author{
MARGARET A. STEVENS, ${ }^{1}$ KEVIN A. GROSSKLAUS, ${ }^{1}$ \\ JOHN H. MCELEARNEY, ${ }^{1}$ and THOMAS E. VANDERVELDE ${ }^{1,2}$ \\ 1.-Renewable Energy and Applied Photonics Labs, ECE Department, Tufts University, Medford, \\ MA 02155, USA. 2.—e-mail: tvanderv@ece.tufts.edu
}

\begin{abstract}
$\mathrm{GaAs}_{1-x} \mathrm{Bi}_{x}$ has been grown by solid-source molecular beam epitaxy using varying substrate rotation rates. Changes in local bismuth saturation were studied by varying the $\mathrm{Bi} / \mathrm{Ga}$ pressure ratio across the wafer. Films were grown on both GaAs and InGaAs buffer layers with varying indium content to change the strain conditions of the bismide layer and the out-of-plane growth rate. All samples demonstrated vertical composition modulations with a period of $\sim 4 \mathrm{~nm}$ that tracked with the rate of growth per substrate rotation cycle. The thermal stability of these composition modulations was shown to behave similarly to bulk GaAsBi. Bismide composition modulations are attributed to the low growth temperature and the varying $\mathrm{Bi} / \mathrm{Ga}$ pressure ratio across the sample rather than the varying V/III ratio.
\end{abstract}

Key words: III-V materials, bismuth compounds, molecular beam epitaxy, film strain, atom probe tomography, substrate rotation

\section{INTRODUCTION}

$\mathrm{GaAs}_{1-x} \mathrm{Bi}_{x}$ is a relatively underdeveloped III-V alloy that opens up new bandgap and lattice constant possibilities for epitaxially grown optoelectronics. Incorporation of small amounts of bismuth (Bi) at group $\mathrm{V}$ sites will reduce the bandgap by as much as $84 \mathrm{meV}$ per \% Bi due to the valence-band anticrossing effect. ${ }^{1,2}$ However, due to the large mismatch in atomic size and electronegativity between arsenic (As) and $\mathrm{Bi}$, the bulk solubility limit of $\mathrm{Bi}$ in $\mathrm{GaAs}$ is extremely low $\left(x<5.2 \times 10^{-5}\right),{ }^{3}$ requiring nonequilibrium growth techniques to access these unique bandgap/lattice constant combinations. Techniques such as molecular beam epitaxy (MBE) and metalorganic vaporphase epitaxy (MOVPE) are well suited for this purpose and have led to bismide devices with $x=0.02$ to $0.074 \mathrm{Bi}$ fraction, as demonstrated in photodiodes $^{4-6}$ and near-infrared (IR) lasers. ${ }^{7}$ Additionally, there is great interest in incorporating

(Received September 29, 2018; accepted January 8, 2019; published online January 31, 2019) higher-bismuth-fraction $(x>0.10)$ materials into devices to reduce Auger recombination in mid-IR lasers ${ }^{8}$ and increase diode efficiency in thermophotovoltaic power systems. ${ }^{9}$

To support the development of GaAsBi-based devices for mid- and far-IR applications, bismuth fractions must be dramatically increased in films without sacrificing material quality. To date, fully strained GaAsBi layers with $x=0.11$ to 0.22 have been grown at very low growth temperatures $\left(<350^{\circ} \mathrm{C}\right)$ and with thicknesses less than $50 \mathrm{~nm}$ to prevent relaxation through defect formation and prevent surface droplet formation. ${ }^{10,11}$ The highest incorporated bismide fractions in GaAsBi have come from growths using near-stoichiometric V/III flux ratios with arsenic dimers $\left(\mathrm{As}_{2}\right)$. However, more recent work has shown that good bismuth incorporation can be achieved under wider ranges of V/III ratios when growing with arsenic tetramers $\left(\mathrm{As}_{4}\right){ }^{12}$ GaAsBi has a tendency to phase separate over a wide range of bismuth fractions ( $x=0.02$ to 0.13 ), resulting in films demonstrating strain-influenced lateral compositional modulations, ${ }^{13}$ coarse-scale vertical phase separation brought on by droplet formation, ${ }^{14}$ or $\mathrm{CuPt}_{\mathrm{B}}$-type atomic ordering. ${ }^{15,16}$ 
Phase separation or spinodal decomposition can yield materials with inconsistent optical and electrical properties, ${ }^{17}$ as well as introduce defects and trap states that degrade performance. ${ }^{18}$ Understanding the mechanisms that lead to phase separation and how separation affects film properties will be critical to creating GaAsBi material suitable for new optoelectronic devices.

To this end, new methods are beginning to be explored to increase bismuth incorporation beyond what can be achieved through conventional tuning of growth parameters. Wood et al. ${ }^{19}$ explored oscillating V/III ratios by rotating their samples slowly through inhomogeneously distributed fluxes in the MBE growth chamber. The resulting samples had a superlattice-like structure, where the maximum bismuth fraction in their rotated samples $(x=0.045)$ exceeded the maximum bismuth fraction achieved in their unrotated samples $(x=0.025)$. They attributed this increase in bismuth to crystallization of gallium $(\mathrm{Ga})$ and $\mathrm{Bi}$ surface clusters that grow and shrink as the sample is rotated through the varying V/III flux ratio. Periodic compositional modulation as a function of substrate rotation has been documented for other MBE-grown materials such as ZnSeTe, ${ }^{20}$ InGaAs, $^{21}$ and AlGaAs. ${ }^{22}$

This work further explores how varying the local $\mathrm{V} / \mathrm{III}$ and $\mathrm{Bi} / \mathrm{Ga}$ ratios via control of sample rotation during growth can impact the saturation point of bismuth in GaAsBi grown on GaAs and InGaAs underlayers and grown with $\mathrm{As}_{4}$ rather than $\mathrm{As}_{2}$. The saturation point is defined as the maximum amount of bismuth that can be incorporated in GaAsBi under specific growth conditions without formation of Ga-Bi surface droplets. Transmission electron microscopy (TEM) paired with atom probe tomography (APT) shows that the bismuth fraction in a nominally $x=0.0655$ film can swing by as much as $\Delta x=0.018$ off the wafer center of rotation, which is a $27 \%$ relative change compared with the film average bismuth fraction. These vertical composition modulations (VCMs) induce local bismuth fractions that exceed the maximum average bismuth fractions achieved in this work. The resulting composition modulations create a vertical "spiral" through the film that follows the rotation of the growing GaAsBi film, such that opposite sides of the wafer have inverse patterns of high-bismuth and low-bismuth stripes. By growing on GaAs and InGaAs underlayers, the out-of-plane lattice constant of the bismide films is varied to alter the period of the VCMs by altering the effective out-ofplane growth rate. Additionally, the thermal stability of VCMs is investigated, as the dramatic swings in bismuth over one rotation rate are linked to the low growth temperature and corresponding lower mobility of $\mathrm{Bi}$ atoms. Ultimately, VCMs in this work are attributed to local changes in the $\mathrm{Bi} / \mathrm{Ga}$ beam equivalent pressure (BEP) ratio across the sample rather than local variations in the V/III ratio, as growing with $\mathrm{As}_{4}$ as opposed to $\mathrm{As}_{2}$ should decrease sensitivity to changes in the V/III ratio.

\section{EXPERIMENTAL PROCEDURES}

All III-V films were grown on a Veeco GENxplor MBE system using a valved As cracker and solidsource effusion cells for the group III elements and $\mathrm{Bi}$. All layers were grown on quarter pieces of 2-inch (001)-oriented epi-ready GaAs substrates and rotated at 2 revolutions per minute (RPM) during film growth. Temperature was measured at all steps by band-edge thermometry using a k-Space Associates BandiT system. Before growth, the GaAs substrates were heated to $620^{\circ} \mathrm{C}$ under $\mathrm{As}_{4}$ overpressure to remove the native oxide, which was confirmed by an increase in brightness in the reflection high-energy electron diffraction pattern (RHEED). After oxide removal, a 500-nm GaAs buffer was grown at $580^{\circ} \mathrm{C}$ at $1 \mathrm{ML} / \mathrm{s}$ (monolayer/second). When used, additional InGaAs buffer layers were grown at a growth rate of $0.5 \mathrm{ML} / \mathrm{s}$ to $0.6 \mathrm{ML} / \mathrm{s}$ at $480^{\circ} \mathrm{C}$ to a thickness of $500 \mathrm{~nm}$ with the indium flux varied to produce the desired composition. To prepare for $\mathrm{GaAsBi}$ growth, samples were cooled to $250^{\circ} \mathrm{C}$ at $30^{\circ} \mathrm{C} / \mathrm{min}$, and the surface presented a $\mathrm{c}(4 \times 4)$ reconstruction for GaAs buffers and a $(4 \times 3)$ reconstruction for InGaAs buffers. GaAsBi layers were grown to thicknesses of $100 \mathrm{~nm}$ to $250 \mathrm{~nm}$ at rates of $0.45 \mathrm{ML} / \mathrm{s}$ to $0.5 \mathrm{ML} / \mathrm{s}$ with a $\mathrm{Bi} / \mathrm{Ga} \mathrm{BEP}$ ratio ranging from 0.27 to 0.29 and an $\mathrm{As}_{4} / \mathrm{Ga} \mathrm{BEP}$ of $\sim 13$.

Bismuth content was examined by high-resolution x-ray diffraction (XRD) (004) and (224) $2 \theta-\Omega$ line scans and (224) reciprocal-space maps collected using a Bruker D8 x-ray diffractometer. Bi content was determined using the Bruker Leptos simulation software, assuming a GaBi lattice constant of $6.33 \AA$ and GaAsBi elastic constants matching GaAs. ${ }^{1}$ These composition measurements were verified by measurements of the GaAsBi film bandgap taken using a variable-angle spectroscopic ellipsometer (VASE, J.A. Woollam Co.). Fundamental optical properties were translated to absorption coefficient as a function of wavelength, and the Tauc approximation was used to determine the band edge of these direct-bandgap layers. ${ }^{23}$ This was translated into an expected bismuth fraction by comparison with previous measurements in literature of bandgap as a function of composition by optical transmission and photoluminescence spectroscopy for compressively strained GaAsBi. ${ }^{11}$

Surface roughness was characterized by atomic force microscopy (AFM) on an Asylum Cypher S. Interface defects $(\mathbf{g}=220$ imaging condition) and vertical compositional variation $(\mathbf{g}=002$ imaging condition) were explored by transmission electron microscopy (TEM) using a JEOL $2010 \mathrm{~F}$ and a JEOL $200 \mathrm{~F}$ ARM operated at $200 \mathrm{kV}$. TEM samples were cross-sections taken along [110] and [110] directions prepared by a focused ion beam (FIB) lift-out method on a FEI Helios 660 dual-beam FIB/SEM 
system. Scanning TEM (STEM) on a JEOL 200F ARM equipped with a high-angle annular detector for dark-field (HAADF) imaging was also used to analyze samples. Additionally, atom probe tomography (APT) was conducted using a Cameca LEAP 4000X HR to analyze the local bismuth composition and determine the degree of compositional oscillations through film thickness.

\section{RESULTS AND DISCUSSION}

To increase the bismuth content to the point of saturation, the $\mathrm{Bi} / \mathrm{Ga} \mathrm{BEP}$ was increased in subsequent samples while keeping all other conditions $\left(\mathrm{As}_{4} / \mathrm{Ga} \mathrm{BEP}\right.$, growth temperature, final thickness, and rotation rate) constant. Figure 1 demonstrates a linear relationship between the $\mathrm{Bi} / \mathrm{Ga} \mathrm{BEP}$ and bismuth fraction as measured by XRD and confirmed by VASE. The surface of the GaAsBi films has roughness $<2 \mathrm{~nm}$ root mean square (RMS) and long diamond-like mounds that are not present in GaAs films grown at the same, low temperatures. Samples with a linear relationship between $\mathrm{Bi} / \mathrm{Ga}$ BEP and bismuth fraction demonstrated $(1 \times 3)$ RHEED surface reconstruction during growth. However, after $\sim x=0.0655$, a drop-off in bismuth incorporation occurred even as the $\mathrm{Bi} / \mathrm{Ga} \mathrm{BEP}$ ratio continued to be increased. Along with this drop-off in composition, a surface reconstruction change to $(2 \times 1)$ occurred, followed by rapid dimming of the RHEED pattern as the growth continued. Ex situ AFM analysis of the surface showed significant roughening, in addition to large droplet formation.

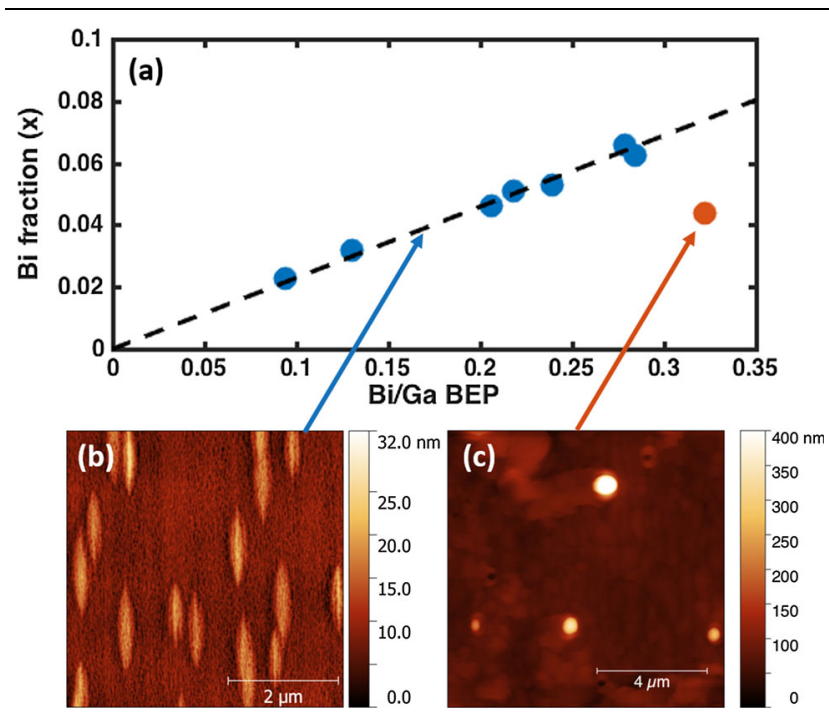

Fig. 1. (a) Bismuth fraction $(x)$ measured by XRD as a function of $\mathrm{Bi} /$ Ga BEP, showing a linear relationship (blue circles) up to a saturation point, after which large $\mathrm{Bi}-\mathrm{Ga}$ surface droplets inhibit $\mathrm{Bi}$ incorporation (orange circle). (b) AFM image of typical surface in the presaturation region. Diamond-like mounds are attributed to roughness caused by defects at the GaAsBi-GaAs interface. (c) AFM image of a typical surface in the postsaturation region. Additional bismuth surface segregates, causing large-scale droplets and significant roughening of the growth surface as the droplets migrate (Color figure online).
This indicates $\mathrm{Bi} / \mathrm{Ga}=0.27$ to 0.29 to be the saturation point for bismuth incorporation under the outlined growth conditions, as beyond this ratio any excess bismuth does not incorporate and instead forms Ga-Bi droplets on the surface, similar to what has been shown for unoptimized Bi incorporation in $\mathrm{GaAsBi}^{24,25}$ and GaSbBi. ${ }^{26}$ To incorporate more bismuth, another growth condition would have to be changed. This could include lowering the growth temperature, ${ }^{10}$ increasing the growth rate, ${ }^{27}$ or changing the V/III ratio (although this has been shown to be less important for growth with $\mathrm{As}_{4}{ }^{12}$ ). Although these methods can increase bismuth incorporation, they have also been shown to have detrimental impacts on crystalline quality and radiative efficiency. ${ }^{28}$

The composition of the GaAsBi layers was determined using two different techniques: XRD and spectroscopic ellipsometry. Figure 2a shows the $2 \theta-\Omega$ XRD 004 line scan and 224 reciprocalspace map of the $\mathrm{GaAsBi}_{0.066}$ sample. Samples for analysis by other methods were grown as thick as $250 \mathrm{~nm}$, but fully strained layers (less than $130 \mathrm{~nm}$ ) were used to determine bismuth content by XRD analysis, as film strain relaxation in thicker films caused loss of Pendellösung fringes and complicated modeling of the XRD data. Spectroscopic ellipsometry was used to confirm bismuth fraction by measuring the absorption edge of our material, as shown in Fig. 2b. This absorption edge was translated into a bismuth fraction by comparison with other tabulations of bandgap as a function of bismuth composition in literature. ${ }^{8,11}$
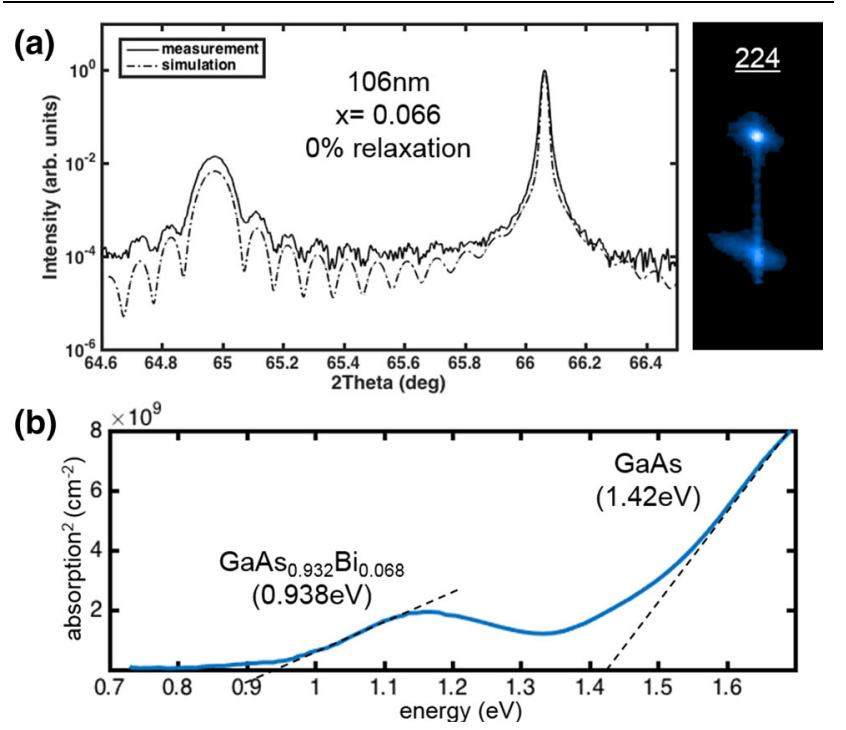

Fig. 2. (a) XRD $2 \theta-\Omega 004$ line scan and 224 reciprocal-space map of $\mathrm{GaAsBi}_{0.066}$ fully strained on a GaAs substrate. Simulation of the data using Leptos software estimates a thickness of $106 \mathrm{~nm}$ and bismuth fraction of $x=0.066$. (b) Ellipsometry data from the same sample showing the GaAsBi absorption edge used to estimate bismuth fraction. 

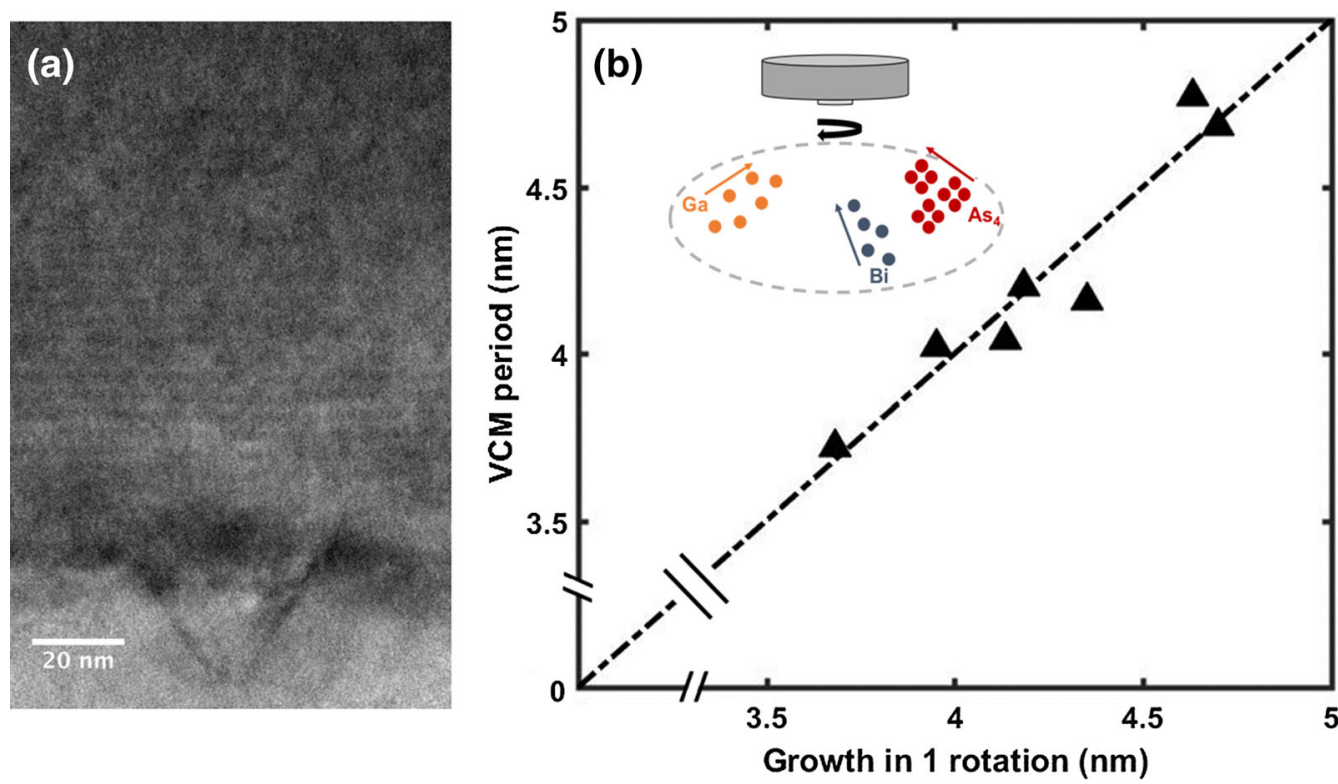

Fig. 3. (a) TEM micrograph taken aligned to the [110] zone axis of $\mathrm{GaAsBi}_{0.066}$ film grown at rotation of 2 RPM, resulting in VCMs along the growth direction. Defects formed at the GaAsBi-GaAs interface lead to roughening of the surface, as seen in AFM images (Fig. 1b). (b) Period of VCM as a function of growth over one rotation through the chamber. The inset schematic shows the relative position of sources in the MBE chamber leading to an inhomogeneous flux at the edges of the quarter wafer.

Figure 3a shows a TEM micrograph taken aligned to the [110] zone axis of a $\mathrm{GaAsBi}_{0.066}$ sample with VCMs aligned to the growth direction. The $\mathrm{GaAsBi}_{0.066} / \mathrm{GaAs}$ structure has a period of compositional oscillation of $\sim 4 \mathrm{~nm}$ and linear defects at the interface between the two materials that may lead to the surface features shown in Fig. 1b. Using the FIB lift-out method for TEM lamella preparation, all TEM specimens were extracted from the same off-center location of the quarter wafers on which the samples were grown. This results in samples that demonstrate the impact of the extreme cases of varying $\mathrm{Bi} / \mathrm{Ga}$ and V/III flux ratios as it rotates through the chamber. Relative locations of the elemental cells with respect to the sample are shown in the inset of Fig. 3b. All samples in this study were grown at 2 RPM, which corresponds to a growth rate of $\sim 4 \mathrm{~nm} /$ revolution depending on the out-of-plane lattice constant and effective growth rate in the (001) direction. While not shown in this work, samples grown under matched conditions with a rotation rate of $20 \mathrm{RPM}$, corresponding to $\sim 0.4 \mathrm{~nm} /$ revolution, do not demonstrate VCMs.

To test the effect of film strain on the formation of these composition oscillations, $\mathrm{GaAsBi}_{0.066}$ films were grown on InGaAs buffer layers where the indium content was varied between samples to change the starting strain conditions of the GaAsBi top layer. GaAsBi was grown to $250 \mathrm{~nm}$ on top of the InGaAs, and was fully strained as confirmed by 224 XRD reciprocal-space maps (not shown). By changing the strain conditions of the GaAsBi layer, the out-of-plane lattice constant was effectively varied, and thereby the film thickness grown over one rotation. Figure $3 \mathrm{~b}$ plots the VCM period compared with the film thickness increase over one sample rotation. A 1:1 relationship was found between these two quantities, demonstrating that VCMs are directly related to the growth rate over one period of sample rotation. Changing the starting strain conditions of the GaAsBi layer did not impact the resulting periodicity of the composition modulations.

To explore the impact of these composition modulations further, $\mathbf{g}=002$ bright-field TEM imaging was used to directly image regions of high bismuth content (dark contrast) and low bismuth content (light contrast). HAADF STEM with energy-dispersive $\mathrm{x}$-ray spectroscopy (not shown) confirms that the changes in TEM image contrast are due to bismuth content and not a combination of bismuth content and local lattice strain. Figure 4 shows TEM images of the same quarter wafer piece, but from opposite off-center locations. The resulting images show that the pattern of high-bismuthcontent and low-bismuth-content regions is different across the sample. At opposite ends of the wafer, the pattern is inverted, as that section of the wafer is rotated through the same flux at different times during the rotation period. The chamber configuration shown in Fig. $3 \mathrm{~b}$ produces the different flux ratios across the sample as it is rotated, resulting in a "spiral" of high bismuth content and low bismuth content through the GaAsBi layer.

To explore further the effects of temperature on bismuth compositional variations, as-grown $\mathrm{GaAsBi}$ samples with rotation-induced VCMs were annealed ex situ in a rapid thermal processor. As bismide materials must be grown below 


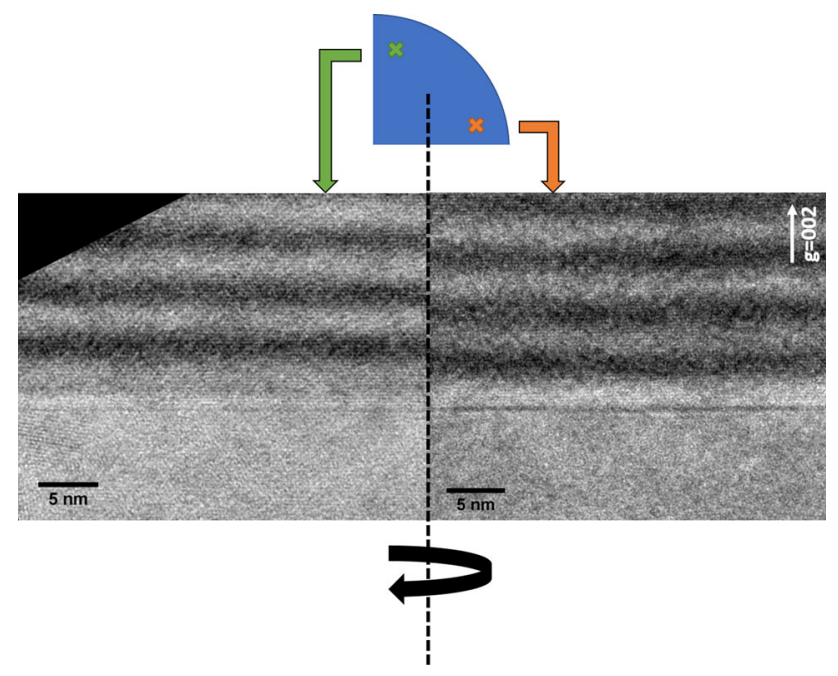

Fig. 4. $\mathbf{g}=002$ bright-field TEM images of $\mathrm{GaAsBi}_{0.066}$ grown compressively strained on relaxed $\ln _{0.08} \mathrm{GaAs} / \mathrm{GaAs}$ buffer layers. The two TEM images represent cross-sectional samples taken from different points on the same quarter two-inch wafer. The stripes on opposite sides of the wafer are the inverse of each other, as each location on the substrate incorporates more bismuth at different times as it rotates through the chamber. No composition modulations were seen in the InGaAs underlayer, grown at $480^{\circ} \mathrm{C}$.

temperatures ideal for most III-V materials, extensive work in literature has documented the effects of annealing GaAsBi both ex situ in rapid thermal processors $^{29-31}$ and in situ after growth ${ }^{32,33}$ to simulate the effects of subsequent device layer growth. Previous research has found that bulk $\mathrm{GaAsBi}$ is fairly stable up to temperatures of $700^{\circ} \mathrm{C}$ to $800^{\circ} \mathrm{C}$, above which bismuth diffusion begins to occur. ${ }^{29}$ This diffusion often takes the form of local bismuth clustering in the GaAsBi layers, leading to metalrich recombination zones. ${ }^{30,31}$ This has been found both for bulk GaAsBi films ${ }^{30,31}$ as well as GaAsBi multiple quantum wells with periods of $\sim 30 \mathrm{~nm} .^{32}$ To investigate the thermal stability of VCMs, the $\mathrm{GaAsBi}_{0.066}$ sample shown in Fig. 4 was annealed in a rapid thermal processor at $700^{\circ} \mathrm{C}$ for $60 \mathrm{~s}$. The sample was annealed in a nitrogen environment and was capped with a GaAs wafer to prevent arsenic outdiffusion. Subsequently, a TEM crosssection was prepared from a location close to the original TEM sample site. The resulting morphology is shown in Fig. 5, clearly showing the presence of large $\mathrm{Bi}$ clusters in the GaAsBi film. High-resolution TEM (not shown) shows that the clusters closely resemble zincblende and rhombohedral $\mathrm{Bi}$ clusters documented in literature. ${ }^{31}$ Residual contrast from the VCMs is still present in the $\mathbf{g}=002$ diffraction image, however it is significantly obscured by the large bismuth droplets. Therefore, films with VCMs are no more thermally stable or resilient to $\mathrm{Bi}$ cluster formation than bulk $\mathrm{GaAsBi}$ films or quantum well structures.

Finally, to investigate the effects of rotation on local bismuth composition, APT needles were extracted from a similar off-rotation center location

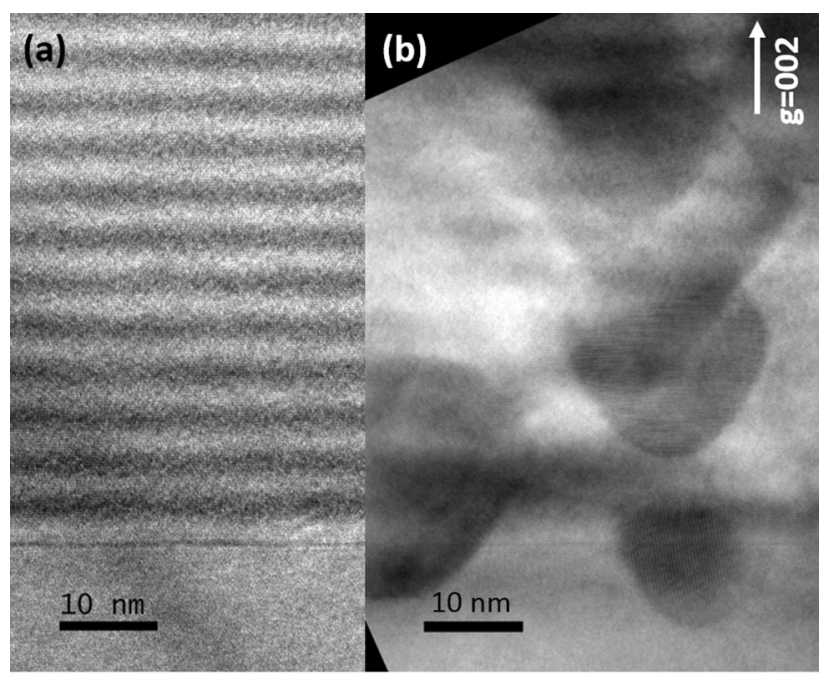

No heat treatment

$700{ }^{\circ} \mathrm{C}$ for 60 seconds

Fig. 5. $\mathbf{g}=002$ TEM images of $\mathrm{GaAsBi}_{0.066} / \mathrm{In}_{0.08} \mathrm{GaAs} / \mathrm{GaAs}$ before (a) and after (b) rapid thermal annealing at $700^{\circ} \mathrm{C}$ for $60 \mathrm{~s}$.

affected by nonuniform flux profiles. A GaAsBi film was grown with a carefully varied rotation rate to investigate composition as a function of growth rotation rate. For the first $\sim 80 \mathrm{~nm}$ of growth, the sample was kept stationary and only rotated every $20 \mathrm{~nm}$ to check the RHEED reconstruction pattern. After $80 \mathrm{~nm}$ of growth, the sample was rotated at $2 \mathrm{RPM}$ for the remainder of the layer thickness, $250 \mathrm{~nm}$ total. Figure 6 shows the reconstruction of the bismuth atoms in the APT needle aligned along the growth direction (001). A line profile of bismuth fraction along the growth direction is also shown. The two regions of rotation have very different average bismuth contents at this off-rotation center location. The first regime, where the sample was kept stationary, has an average bismuth fraction close to the composition indicated by XRD and VASE ( $x=0.067$, as shown in Fig. 2$)$. However, the region with continuous rotation at $2 \mathrm{RPM}$ has a decreased average bismuth fraction $(x=0.054)$. The average, minimum, and maximum bismuth fractions for the two rotation regions are shown in Table I. In determining these values, the first $2 \mathrm{~nm}$ and last $15 \mathrm{~nm}$ of the film thickness were excluded to eliminate near GaAs interface and film surface effects and the effects of ion damage at the edges of the APT needle.

The results in Table I demonstrate that the bismuth fraction in the region grown with no rotation was higher in all cases (maximum, minimum, average) compared with the region grown with rotation at 2 RPM. This indicates that the sample was extracted from a wafer region that was grown with the most favorable conditions for bismuth incorporation, i.e., lowest $\mathrm{As}_{4} / \mathrm{Ga} \mathrm{BEP}$ or highest Bi flux. This is different from the results found by Wood et al., ${ }^{19}$ who used intensity analysis from STEM images paired with XRD results to 


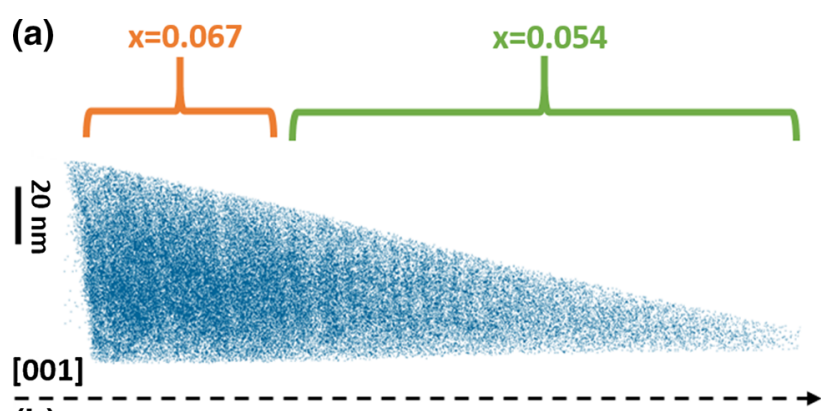

$\overline{(b)}$

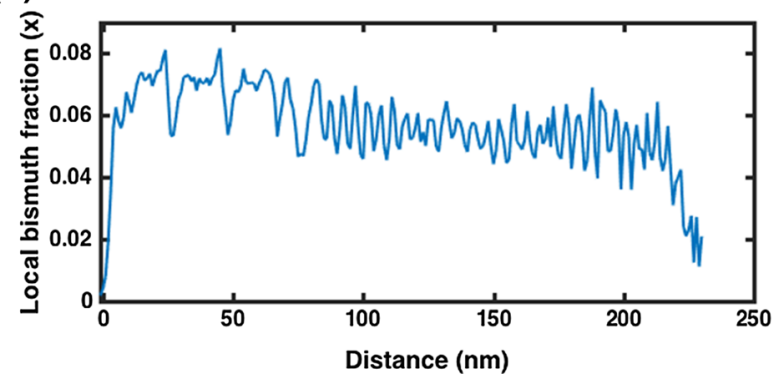

Fig. 6. APT data taken from an off-center location in the $\mathrm{GaAsBi}_{0.066}$ layer. (a) Reconstruction of the sample needle shows bismuth location as a function of depth (blue), demonstrating the two regions of rotation. (b) Local bismuth fraction plotted as a function of depth for analysis of maximum, minimum, and average bismuth content in the two rotation regions (Color figure online).

Table I. Local bismuth fraction as a function of rotation rate from an off-center location

\begin{tabular}{|c|c|c|c|}
\hline Rotation & Average & Minimum & Maximum \\
\hline 0 RPM & 0.067 & 0.046 & 0.082 \\
\hline $2 \mathrm{RPM}$ & 0.054 & 0.036 & 0.072 \\
\hline
\end{tabular}

determine bismuth content and found that bismide fractions in off-center locations were significantly higher when rotated than when kept stationary. They attributed this effect to growth and shrinkage of $\mathrm{Bi} / \mathrm{Ga}$ clusters as they rotated through regions of high and low $\mathrm{As}_{2} / \mathrm{Ga}$ flux ratio. Previous work in literature has shown that $\mathrm{GaAsBi}$ grown with $\mathrm{As}_{4}$ is less sensitive to V/III ratio, ${ }^{12}$ indicating that the $\mathrm{Bi}$ content in GaAsBi films grown in this work using $\mathrm{As}_{4}$ may be less sensitive to V/III ratio. Therefore, VCMs produced in this work are likely not caused by variation in V/III ratio with rotation, but instead are due to variation in $\mathrm{Bi}$ flux. This leads to an increase in $\mathrm{Bi}$ content that continues to follow the linear trend shown in Fig. 1a despite rotationinduced changes. When the off-center region of the sample is stationary in front of the high-Bi-flux region of the chamber, a surface layer of bismuth can stabilize and lead to increased incorporation. ${ }^{10}$ When it is rotated through the high-Bi-flux region, the bismuth has less time to stabilize on the surface and incorporation suffers. A bismuth soaking step to initiate good surface coverage of Bi before initiating GaAsBi growth could eliminate this problem, however it would need to be carefully optimized so as not to induce large $\mathrm{Bi}$ droplets. ${ }^{34,35}$

\section{CONCLUSIONS}

Vertical composition modulations in $\mathrm{GaAsBi}_{0.066}$ structures were analyzed as a function of starting strain conditions and rotation rate. Growing on InGaAs buffer layers to change the strain conditions and effective growth rate did not impact the period of the composition modulations, as they still followed a 1:1 relationship with the rotation rate. VCMs annealed at $700^{\circ} \mathrm{C}$ for $60 \mathrm{~s}$ resulted in $\mathrm{Bi}$ diffusion into large $\mathrm{Bi}$-rich clusters, similar to what has been shown for bulk GaAsBi as well as quantum well structures. Local bismuth content in off-center locations was higher in the samples grown without rotation than those grown with rotation, in contrast to previous work in literature. This discrepancy is attributed to growing with $\mathrm{As}_{4}$ rather than $\mathrm{As}_{2}$, as GaAsBi grown with arsenic tetramers tends to be less sensitive to V/III ratio.

\section{ACKNOWLEDGMENTS}

This work is supported by the Office of Naval Research (N00014-15-1-2946) and the National Science Foundation (NSF ECCS-1337783). M. Stevens acknowledges support from the NASA Space Technology Research Fellowship (NNX15AQ79H). This work was performed in part at the Center for Nanoscale Systems (CNS), a member of the National Nanotechnology Coordinated Infrastructure Network (NNCI), which is supported by the National Science Foundation under NSF Award No. 1541959. CNS is part of Harvard University. This work also made use of the Shared Experimental Facilities at the Massachusetts Institute of Technology, supported in part by the MRSEC Program of the National Science Foundation under Award No. DMR - 1419807.

\section{CONFLICT OF INTEREST}

The authors declare that they have no conflict of interest.

\section{OPEN ACCESS}

This article is distributed under the terms of the Creative Commons Attribution 4.0 International License (http://creativecommons.org/licenses/by/4.0/), which permits unrestricted use, distribution, and reproduction in any medium, provided you give appropriate credit to the original author(s) and the source, provide a link to the Creative Commons license, and indicate if changes were made.

\section{REFERENCES}

1. S. Tixier, M. Adamcyk, T. Tiedje, S. Francoeur, A. Mascarenhas, P. Wei, and F. Schiettekatte, Appl. Phys. Lett. 82, 2245 (2003). 
2. K. Alberi, J. Wu, W. Walukiewicz, K.M. Yu, O.D. Dubon, S.P. Watkins, C.X. Wang, X. Liu, Y.J. Cho, and J. Furdyna, Phys. Rev. B 75, 045203 (2007).

3. H. Jacobsen, B. Puchala, T.F. Kuech, and D. Morgan, Phys. Rev. B 86, 085207 (2012).

4. R.D. Richards, A. Mellor, F. Harun, J.S. Cheong, N.P. Hylton, T. Wilson, T. Thomas, J.S. Roberts, N.J. Ekins-Daukes, and J.P.R. David, Sol. Energy Mater. Sol. Cells 172, 238 (2017).

5. Z. Zhou, D.F. Mendes, R.D. Richards, F. Bastiman, and J.P. David, Semicond. Sci. Technol. 30, 094004 (2015).

6. A. Geižutis, V. Pačebutas, R. Butkute, P. Svidovsky, V. Strazdiene, and A. Krotkus, Solid State Electron. 99, 101 (2014).

7. I.P. Marko, S.R. Jin, K. Hild, Z. Batool, Z.L. Bushell, P. Ludewig, W. Stolz, K. Volz, R. Butkute, V. Pačebutas, A. Geizutis, A. Krotkus, and S.J. Sweeney, Semicond. Sci. Technol. 30, 094008 (2015).

8. Z. Batool, K. Hild, T.J.C. Hosea, T. Tiedje, and S.J. Sweeney, J. Appl. Phys. 111, 113108 (2012).

9. M. Stevens, A. Licht, N. Pfiester, E. Carlson, K. Grossklaus, and T. Vandervelde, in 44th IEEE Photovoltaics Specialist Conference Proceedings (2017), pp. 0701-0705.

10. R.B. Lewis, M. Masnadi-Shirazi, and T. Tiedje, Appl. Phys. Lett. 101, 082112 (2012).

11. M. Masnadi-Shirazi, R.B. Lewis, V. Bahrami-Yekta, T. Tiedje, M. Chicoine, and P. Servati, J. Appl. Phys. 116, 223506 (2014).

12. F. Bastiman, A.R.B. Mohmad, J.S. Ng, J.P.R. David, and S.J. Sweeney, J. Cryst. Growth 338, 57 (2012).

13. E. Luna, M. Wu, J. Puustinen, M. Guina, and A. Trampert, J. Appl. Phys. 117, 185302 (2015).

14. C.R. Tait, L. Yan, and J.M. Millunchick, Appl. Phys. Lett. 111, 042105 (2017).

15. A.G. Norman, R. France, and A.J. Ptak, J. Vac. Sci. Technol. B 29, 03C121 (2011).

16. D.F. Reyes, F. Bastiman, C.J. Hunter, D.L. Sales, A.M Sanchez, J.P.R. David, and D. González, Nanoscale Res. Lett. 9, 23 (2014).

17. L.C. Hirst, M.P. Lumb, J. Abell, C.T. Ellis, J.G. Tischler, I. Vurgaftman, J.R. Meyer, R.J. Walters, and M. González, J. Appl. Phys. 117, 215704 (2015).
18. I. Garcia, I. Rey-Stolle, C. Algora, W. Stolz, and K. Volz, J. Cryst. Growth 310, 5209 (2008).

19. A.W. Wood, S.E. Babcock, J. Li, and A.S. Brown, J. Vac. Sci. Technol. A Vac. Surf. Film 33, 031506 (2015).

20. P. Reimer, J.R. Buschert, S. Lee, and J. Furdyna, Phys. Rev. 61, 8388 (2000).

21. K. Alavi, P.M. Petroff, W.R. Wagner, and A.Y. Cho, J. Vac. Sci. Technol. B 1, 146 (1983).

22. S.N.G. Chu, N. Chand, D.L. Sivco, and A.T. Macrander, J. Appl. Phys. 65, 3838 (1989).

23. J.I. Pankove, Optical Processes in Semiconductors (New York: Dover, 1975).

24. G. Vardar, S.W. Paleg, M.V. Warren, M. Kang, S. Jeon, and R.S. Goldman, Appl. Phys. Lett. 102, 042106 (2013).

25. E. Sterzer, N. Knaub, P. Ludewig, R. Straubinger, A. Beyer, and K. Volz, J. Cryst. Growth 408, 71 (2014).

26. A. Duzik and J.M. Millunchick, J. Cryst. Growth 390, 5 (2014).

27. A.J. Ptak, R. France, D.A. Beaton, K. Alberi, J. Simon, A. Mascarenhas, and C.S. Jiang, J. Cryst. Growth 338, 107 (2012).

28. J. Li, K. Forghani, K. Collar, T.F. Kuech, A.S. Brown, W. Jiao, and W. Kong, J. Appl. Phys. 116, 043524 (2014).

29. A.R. Mohmad, F. Bastiman, C.J. Hunter, R. Richards, S.J. Sweeney, J.S. Ng, and J.P.R. David, Appl. Phys. Lett. 101, 012106 (2012).

30. J. Puustinen, M. Wu, E. Luna, A. Schramm, P. Laukkanen, M. Laitinen, T. Sajavaara, and M. Guina, J. Appl. Phys. 114, 243504 (2013).

31. M. Wu, E. Luna, J. Puustinen, M. Guina, and A. Trampert, Nanotechnology 25, 205605 (2014).

32. A.W. Wood, W. Chen, H. Kim, and Y. Guan, Nanotechnology 28, 215704 (2017).

33. H. Kim, K. Forghani, Y. Guan, K. Kim, A.W. Wood, J. Lee, S.E. Babcock, T.F. Kuech, and L.J. Mawst, J. Cryst. Growth 452, 276 (2016).

34. R.D. Richards, F. Bastiman, C.J. Hunter, D.F. Mendes, A.R Mohmad, J.S. Roberts, and J.P.R. David, J. Cryst. Growth 390,120 (2014).

35. F. Bastiman, A.G. Cullis, J.P.R. David, and S.J. Sweeney, J. Cryst. Growth 341, 19 (2012). 